\title{
Protée
}

\section{Cinéma et intermédialité}

\section{Denis Bellemare}

Volume 28, numéro 3, 2000

Mélancolie entre les arts

URI : https://id.erudit.org/iderudit/030607ar

DOI : https://doi.org/10.7202/030607ar

Aller au sommaire du numéro

Éditeur(s)

Département des arts et lettres - Université du Québec à Chicoutimi

ISSN

0300-3523 (imprimé)

1708-2307 (numérique)

Découvrir la revue

Citer ce compte rendu

Bellemare, D. (2000). Compte rendu de [Cinéma et intermédialité]. Protée, 28(3),

85-87. https://doi.org/10.7202/030607ar d'utilisation que vous pouvez consulter en ligne.

https://apropos.erudit.org/fr/usagers/politique-dutilisation/ 


\title{
compte rendu critique
}

\section{Cinéma et intermédialité}

\author{
par Denis Bellem are
}

\begin{abstract}
'étonnante somme encyclopédique que peut représenter ce numéro de la revue d'études cinématographiques CiNémAS (vol.10, nos 2-3, printemps 2000) ne pourra que saisir le lecteur par sa richesse. Chaque article s'avère un champ de recherches en soi, tant son objet d'études est spécifique, tant chaque texte construit son propre paradigme d'agencements à la lumière de réflexions philosophiques, historiques, esthétiques bien délimitées et fort diverses. Cette multiplicité des lieux d'intérêt (télévision, cinéma, littérature) et des champs disciplinaires du savoir, certes constitutive de ce concept de l'entre-deux de l'intermédialité, amènera le lecteur à prioritairement s'interroger non pas sur la qualité d'un tel projet mais plutôt sur sa possible étendue et son effective portée. II s'agit certes de tracer des lignes et non des points fixes de recherche, comme l'exprime Germain Lacasse; il ne faut pas non plus, nous le comprenons bien à la suite de l'article de Jürgen E. Müller, construire un système fermé, un système de systèmes, mais alors quelles seront les perspectives privilégiées, s'il y a lieu, par l'axe intermédiatique, quels sont les divers degrés de pertinence de ces axes de recherches? C'est ce que nous aurons parfois de la difficulté à mesurer dans la vaste entreprise de ce numéro.
\end{abstract}

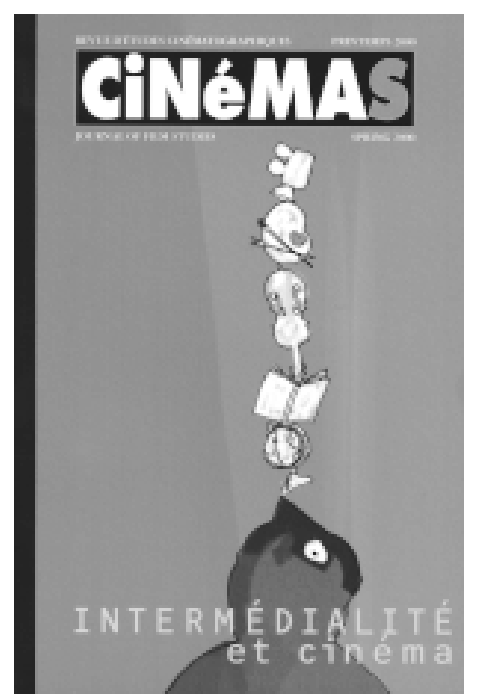

Chaque article mériterait en soi un compte rendu, nous favoriserons cependant une approche générale permettant de mieux saisir les territoires et/ou les déterritorial isations de l'intermédialité. Cela appelle une question de géographie conceptuelle. 0 ù se situe le concept d'intermédialité en regard de l'interdisciplinarité, de l'intertextualité, de l'interartialité, de l'interactivité - à côté? autour? au-dessus?-, pour dire leurs échanges, leurs nécessaires réciprocités et leurs incomplétudes?

La présentation par Silvestra M arinello module bien l'entrée en lecture de ce numéro en nous invitant à saisir la dynamique entre les pratiques signifiantes qu'appelle la perspective inter- 
médiatique. L'objectif du numéro n'est pas de définir l'intermédialité, écritelle, ni d'en fairel'historique, soit, mais heureusement un article fondamental nous cible stratégiquement certaines perspectives théoriques comblant en cela notre entre-savoir et notre entrepercevoir de l'intermédialité.

«L'intermédialité, une nouvelle approche interdisciplinaire» de Jürgen $E$. Müller se trouve au cœur et au centre (p. 105-134) de ce numéro. Dans la recherche de son axe de pertinence pour mieux saisir la dynamique intermédiatique, l'auteur travaille en une sorte d'inclusions réciproques, nécessaires et complémentaires, les divers concepts d'interdisciplinarité, d'intertextualité - «le passage d'un système de signes à un autre » - et d'intermédial ité - «les processus de production de sens liés à des interactions médiatiques». Les additions de concepts, allant de la fonction de l'hétérotopie de Foucault à l'interstice de Deleuze, aux lieux de passage de Benjamin, à la détermination de l'«entre » de Bellour, obligent le regard historique; I'archéologie de la modernité et de la postmodernité ne doit pas oublier les apports du Q uattrocento italien, de sa Renaissance.

Dans une perspective plus contemporaine, la description des quatre types d'intermédialité chez Jens Schröter (p. 107) nous éclaire: I'intermédialité synthétique de la fusion de plusieurs médias dans un intermédia, l'intermédialité formelle, l'intermédialité transformationnelle de différents médias dans un autre média, l'intermédialité ontologique comme processus toujours présent dans les médias. Cette plasticité conceptuelle chez M üller ne se joue pas théoriquement comme en certains autres articles d'oppositions concurrentes et opposantes entre modernité et postmodernité (l'intermédialité et sa pensée ne sont-elles que postmodernes?), entre la discursivité filmique chez Metz etl'articulation de la pensée en imageschez Deleuze, telles que posées dans l'article de Marion Froger. Chez Müller, «la conception de l'intermédialité ne néglige pas la question de la différence entre médias», des interactions constantes entre concepts médiatiques et de leurs spécificités multiples; sa conception se nourrit de ce paradoxe même, de son ambivalence constitutive. Cette réorientation des recherches dans les médias pourra prendre cinq voies, cinq axes: de la pragmatique, de la cognition, de la sémiotique, de l'esthétique et de I'histoire des médias et de l'intermédialité.

De ces processus permanents de rapprochements et de différenciation de reterritorialisation virtuelle, Germain Lacasse montre et démontre bien dans son article «Intermédialité, deixis et politique» (p.85-104) la mobilité des agencements des médias entre eux. Ses exemples de cinéma oral, de théâtre cinématographique, de radio visuelle et de cinéma radiophonique, puisés dans l'expérience médiatique du Québec au début du XX $X^{\mathrm{e}}$ siècle, décrivent la sphère intermédiatique comme un «espace à la fois réel et symbolique constitué par les médias et leur rapport avec les communau- tés» (p. 86). Cette sphère s'inscrit dans un espace temporairement nomade, en éternel changement et en perpétuel risque d'institutionnalisation, repoussant sans cesse les échanges entre divers modes de relation et d'expression de l'une et de l'autre des pratiques signifiantes vers de nouvelles, pour être plus tard appelées à devenir anciennes. Cette position de fluctuations et de transformations n'intègre toutefois pas l'idée chez Jens Schröter d'une intermédialité ontologique présente comme processus dans tous les médias. Cet article construit ainsi par défaut deux paradigmes concurrents où l'intermédialité s'instaure comme un moment de changement opposé à son moment d'institutionnalisation, comme la nouveauté de la marge repoussant la norme vers l'ancien. L'intermédialité s'érige-t-elle, voire s'institutionnaliset-elle sur le mythe de la nouveauté à tout prix?

Le texte de Catherine Russel, «L'historiographie parallaxial e et la flâneuse» (p. 151-168), risque ce même type d'approche opposant deux régimes de spectatorat de la modernité et de la postmodernité, du voyeur masculin et de la voyageuse féminine, de I'uniformité du cinéma classique et de I'hétérogénéité des œuvres intermédiatiques. J'ai toujours pensé que la description de la spectatrice de cinéma dans les théories féministes analysait enfin la véritable posture spectatorielle primaire, labile, fluide, multiple audelà des genres. En cours d'écriture de cette historiographie, les théories de Catherine Russell, de M iriam Hansen, d'Anne Friedberg, de Giuliana Bruno 
opèrent d'intéressants glissements déboulonnant de façon nouvelle ces oppositions. Le cinéma classique ne serait somme toute qu'un court moment de I'histoire et, qui plus est, traduisant beaucoup plus une certaine crise des discours le légitimant qu'une aussi essentielle et présupposée opposition. II faudra peut-être penser un jour que ce sont les moments de médialité qui ont été rares dans I'histoire des expressions artistiques et non les moments de l'intermédialité. Sous cet angle, la perte del'aura chez Walter Benjamin ne manifeste pas selon Catherine Russel

[...] une attitude nostalgique pour ce qui disparaît: elle s'en prend plutôt à une conception utopique des transformations historiques. (p. 157)

Est-ce cela que peut évoquer et suggérer I'article de Walter Moser " "Puissance baroque" dans les nouveaux médias» (p. 39-64) ? Cette relative harmonie des divers médias entre eux en un mouvement d'ouverture, de pluralisation et d'intensification? Les divers médias sont-ils des mondes compossibles pouvant coexister en convergence comme dans le film Prospero's Books de Peter Greenaway? L'auteur analyse ce film dans toute la force de ses métamorphoses et ses distinctions conceptuelles entre interartialité et intermédialité ouvrent tout un pan de signification dans leur apport à l'interdisciplinarité.
Si l'interartialité s'intéresse à l'interaction entre divers arts, y compris le passage de l'un à l'autre et la prise en charge de l'un par l'autre, l'intermédialité articule le même type de relation entre deux ou plusieurs médias. La relation interactionnelle est donc la même de part et d'autre; par contre, la relation entre art et média ne montre pas la même symétrie. (p. 44)

L'intermédialité au cinéma et dans les autres arts peut nous aider à comprendre cette identité des contraires: sortir du cinéma et aller ailleurs à condition de ne pas cliver les hétérogénéités et les spécificités en des discours extrêmes, discours souvent soumis à des contradictions cachées. Les divers axes de recherche intermédiatiques appellent divers types d'assemblage par l'établissement de rapports entre les diverses fonctions de divers dispositifs variables, par la redistribution de ces territoires et leur constitution en de nouvelles expressions du monde sensible. Deux articles par leur grâce et leur ouverture nous invitent à cette lecture: «Palomar: intermédialité et archéologie de la vision» d'Antonio Costa (p. 169-184) et «Theoretical Apparitions of H aiku: An Intermedial Interrogation of Modernity » de Tollof N elson (p. 185-203). 